\title{
Clover improvement for stout-smeared $2+1$ flavour SLiNC fermions: non-perturbative results
}

\author{
N. Cundy ${ }^{a}$, M. Göckeler ${ }^{a}$, R. Horsley ${ }^{* b}$, T. Kaltenbrunner ${ }^{a}$, A. D. Kennedy ${ }^{b}$, \\ Y. Nakamura ${ }^{c}$, H. Perlt ${ }^{d}$, D. Pleiter ${ }^{c}$, P. E. L. Rakow ${ }^{e}$, A. Schäfer ${ }^{a}$, G. Schierholz ${ }^{a c f}$, \\ A. Schiller ${ }^{d}$, H. Stüben ${ }^{g}$ and J. M. Zanotti ${ }^{b}$ \\ ${ }^{a}$ Institut für Theoretische Physik, Universität Regensburg, 93040 Regensburg, Germany \\ ${ }^{b}$ School of Physics and Astronomy, University of Edinburgh, Edinburgh EH9 3JZ, UK \\ ${ }^{c}$ John von Neumann Institute NIC / DESY Zeuthen, 15738 Zeuthen, Germany \\ ${ }^{d}$ Institut für Theoretische Physik, Universität Leipzig, 04109 Leipzig, Germany \\ ${ }^{e}$ Theoretical Physics Division, Department of Mathematical Sciences, University of Liverpool, \\ Liverpool L69 3BX, UK \\ ${ }^{f}$ Deutsches Elektronen-Synchrotron DESY, 22603 Hamburg, Germany \\ ${ }^{g}$ Konrad-Zuse-Zentrum für Informationstechnik Berlin, 14195 Berlin, Germany \\ E-mail: rhorsley@ph.ed.ac.uk

\section{QCDSF-UKQCD Collaborations}

\begin{abstract}
We discuss an action in which the fermion matrix has single level stout smearing for the hopping terms together with unsmeared links for the clover term. With the (tree level) Symanzik improved gluon action this constitutes the Stout Link Non-perturbative Clover or SLiNC action. To cancel $O(a)$ terms the clover coefficient, $c_{s w}$ has to be tuned. We present here preliminary results of a non-perturbative determination of $c_{s w}$ using the Schrödinger functional and as a by-product also a determination of the critical hopping parameter, $\kappa_{c}$. A determination of the renormalisation constant for the local vector current is also given. Comparisons of the results are made with lowest order perturbation theory results.
\end{abstract}

The XXVI International Symposium on Lattice Field Theory

July 14-19 July 2008

Williamsburg, USA

\footnotetext{
* Speaker.
} 


\section{1. $O(a)$ Improvement}

When constructing a lattice QCD action, even the simplest gluon Lagrangian action has only $O\left(a^{2}\right)$ corrections. The naive fermion action also has $O\left(a^{2}\right)$ corrections, but suffers from the 'doubling problem' describing 16 flavours in the continuum limit. A 'cure' is to add the Wilson mass term, so 15 flavours decouple in the continuum limit, but the price is that there are now $O(a)$ corrections, so that for example for a ratio of hadron masses

$$
\frac{m_{H}}{m_{H^{\prime}}}=r_{0}+a r_{1}+O\left(a^{2}\right) .
$$

The Symanzik approach is a systematic improvement to $O\left(a^{n}\right)$ (where in practice $n=2$ ) by adding a basis (an asymptotic series) of irrelevant operators and tuning their coefficients to remove completely $O\left(a^{n-1}\right)$ effects. Restricting improvement to on-shell quantities the equations of motion reduce the set of operators in both the action and in matrix elements. Indeed, for $O(a)$ improvement only one additional operator in the action is required

$$
\mathscr{L}_{\text {clover }} \propto a c_{s w} \sum \bar{\psi} \sigma_{\mu \nu} F_{\mu \nu} \psi
$$

the so-called 'clover term'. So if we can improve one on-shell quantity this then fixes $c_{s w}$ as a function of the lattice spacing $a$ or equivalently of $g_{0}^{2}$, so that all other physical on-shell quantities are automatically improved to $O(a)$, i.e., we now have

$$
\frac{m_{H}}{m_{H^{\prime}}}=r_{0}+O\left(a^{2}\right) .
$$

Matrix elements still require additional $O(a)$ operators, for example

$$
\begin{aligned}
\mathscr{A}_{\mu} & =\left(1+b_{A} a m_{q}\right)\left(A_{\mu}+c_{A} a \partial_{\mu}^{L A T} P\right) \\
\mathscr{P} & =\left(1+b_{P} a m_{q}\right) P,
\end{aligned}
$$

with

$$
A_{\mu}=\bar{q} \gamma_{\mu} \gamma_{5} q, \quad P=\bar{q} \gamma_{5} q
$$

An easily determined quantity is the quark mass, determined from the PCAC relation ${ }^{1}$

$$
m_{q}^{W I}=\frac{\left\langle\partial_{0}^{L A T}\left(A_{0}\left(x_{0}\right)+c_{A} a \partial_{0}^{L A T} P\left(x_{0}\right)\right) O\right\rangle}{2\left\langle P\left(x_{0}\right) O\right\rangle} .
$$

Choosing different boundary conditions or operators, $O$, gives different determinations of the quark mass $m_{q}^{W I(i)}, i=1,2$. If the quark mass is improved then its errors are $O\left(a^{2}\right)$. So we can determine improvement coefficients, $c_{s w}, \ldots$, by finding the point where

$$
m_{q}^{W I(1)}=m_{q}^{W I(2)} .
$$

\footnotetext{
${ }^{1}$ This is equivalent to considering the renormalised quark mass $m_{q R}^{W I}=\frac{Z_{A}\left(1+b_{A} a m_{q}\right)}{Z_{P}\left(1+b_{P} a m_{q}\right)} m_{q}^{W I}$ as the difference is just a numerical factor, which in the chiral limit does not effect considerations of $O(a)$-improvement.
} 
The ALPHA Collaboration achieved this by means of the 'Schrödinger functional', [1]. Dirichlet boundary conditions are applied on the time boundaries to the fields. For the gluon fields fixing them on $x_{0}=0$ and $T$ is then equivalent to a constant chromo-electric background field (which means that simulations with $m_{q} \sim 0$ with no zero mode problems are possible), while the fixed quark fields $(\rho, \bar{\rho})$ can be taken as sinks/sources to build operators for correlation functions. For example here we can take at the lower boundary $x_{0}=0(i=1)$ and upper boundary $x_{0}=T(i=2)$

$$
O^{(i)}=\sum_{\vec{y}, \vec{z}} \bar{\rho}^{(i)}(\vec{y}) \gamma_{5} \rho^{(i)}(\vec{z})
$$

So we can investigate PCAC behaviour at different distances from the boundaries. Redefining the quark mass slightly (but in a way which coincides to $O\left(a^{2}\right)$ in the improved theory, [2]) to eliminate the unknown $c_{A}\left(m_{q}^{W I} \rightarrow M\right)$ we can define improvement when

$$
(M, \Delta M)=(0,0)
$$

where

$$
M \equiv M^{(1)} \quad \Delta M \equiv M^{(1)}-M^{(2)},
$$

are chosen at some suitable $x_{0}$, [没]. This gives the required critical $c_{s w}^{*}$ and $\kappa_{c}^{*}$.

There are (small) ambiguities due to the finite volume used. In an infinite volume we expect $O\left(a \Lambda_{Q C D}\right)$ contributions (in the chiral limit, otherwise there are also extra $O\left(a m_{q}\right)$ terms) due to the different boundary conditions or operators chosen. In a finite volume there are additional $O\left(a / L_{S}\right)$ terms. $O\left(a \Lambda_{Q C D}\right) \rightarrow 0$ as $a$ (or $\left.g_{0}^{2}\right) \rightarrow 0$, but $O\left(a / L_{s}\right) \sim O\left(1 / N_{s}\right)$ (where $\left.L_{s}=a N_{s}\right)$. We can either keep $L_{s}$ fixed in physical units as $a \rightarrow 0$ (the 'constant physics condition') so $O\left(a / L_{s}\right) \rightarrow 0$, or alternatively simulate for several values of $N_{s}$ and extrapolate to $N_{s} \rightarrow \infty$. The 'Poor man's solution' is to evaluate at large $\beta \rightarrow \infty$ (small $a$ ) and subtract this result. Practically we have found that for $c_{s w}$ this $O\left(1 / N_{s}\right)$ term is negligible, while for $Z_{V}$, this subtraction is about a $1 \%$ affect.

\section{The SLiNC action}

We shall apply the Schrödinger functional formalism to $2+1$ flavour stout link clover fermions SLiNC fermions (Stout Link Non-perturbative Clover). In a little more detail

$$
\begin{aligned}
S_{F}=\sum_{x}\{ & \kappa \bar{\psi}(x) \tilde{U}_{\mu}(x+\hat{\mu})\left[\gamma_{\mu}-1\right] \psi(x-\hat{\mu})-\kappa \bar{\psi}(x) \tilde{U}_{\mu}^{\dagger}(x-\hat{\mu})\left[\gamma_{\mu}+1\right] \psi(x+\hat{\mu}) \\
+ & \left.\bar{\psi}(x) \psi(x)+\frac{1}{2} c_{s w}\left(g_{0}^{2}\right) \bar{\psi}(x) \sigma_{\mu \nu} F_{\mu \nu}(x) \psi(x)\right\} .
\end{aligned}
$$

The hopping terms (Dirac kinetic term and Wilson mass term) use a once iterated stout smeared link or 'fat link',

$$
\begin{aligned}
\tilde{U}_{\mu} & =\exp \left\{i Q_{\mu}(x)\right\} U_{\mu}(x) \\
Q_{\mu}(x) & =\frac{\alpha}{2 i}\left[V U^{\dagger}-U V^{\dagger}-\frac{1}{3} \operatorname{Tr}\left(V U^{\dagger}-U V^{\dagger}\right)\right],
\end{aligned}
$$

( $V_{\mu}$ is the sum of all staples around $U_{\mu}$ ) while the clover term remains built from 'thin' links - they are already of length $4 a$ and we want to avoid the fermion matrix becoming too extended. Smearing 
is thought to help at present lattice spacings and the stout variation is analytic which means that the derivative can be taken (so the HMC force is well defined) and perturbative expansions are also possible, [3].

To complete the action we also use the Symanzik tree-level gluon action

$$
S_{G}=\frac{6}{g_{0}^{2}}\left\{c_{0} \sum_{\text {Plaquette }} \frac{1}{3} \operatorname{Re} \operatorname{Tr}\left(1-U_{\text {Plaquette }}\right)+c_{1} \sum_{\text {Rectangle }} \frac{1}{3} \operatorname{Re} \operatorname{Tr}\left(1-U_{\text {Rectangle }}\right)\right\},
$$

together with

$$
c_{0}=\frac{20}{12}, c_{1}=-\frac{1}{12} \quad \text { and } \quad \beta=\frac{6 c_{0}}{g_{0}^{2}}=\frac{10}{g_{0}^{2}}
$$

\section{The lattice simulation}

The lattice simulation used the Chroma software library, [丹]. The Schrödinger Functional details follow [5]. All results were generated on $8^{3} \times 16$ lattices using the HMC algorithm. A mild smearing of $\alpha=0.1$ was used. A series of simulations were performed (typically generating $O(3000)$ trajectories), quadratic and then linear interpolations of the $M, \Delta M$ results being used to locate the critical point.

We thus have a two-parameter interpolation in $c_{s w}$ and $\kappa$ which is split here into two separate interpolations. First plotting $\Delta M$ against $M$ and then interpolating to $M=0$ gives $\Delta M\left(c_{s w}, \kappa_{c}\left(c_{s w}\right)\right)$. A typical result is shown in Fig. 1. These values of $\Delta M$ for $M=0$ for various $\beta$ values are then

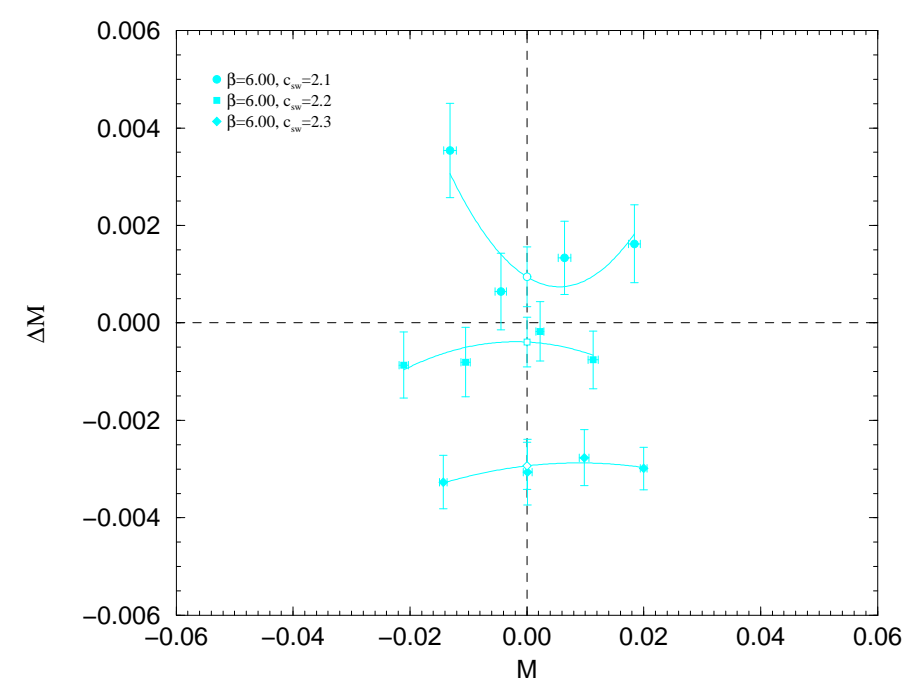

Figure 1: $\Delta M$ against $M$ for $\beta=6.00$ (filled symbols) together with quadratic interpolations to $M=0$ (the open symbols).

plotted against $c_{s w}$ as shown in Fig. \&. $\Delta M=0$ then gives $c_{s w}^{*}$.

A similar procedure yields $\kappa_{c}^{*}$ : plotting $M$ against $1 / \kappa$ and interpolating to $M=0$ gives $\kappa_{c}\left(c_{s w}\right)$. Then subsequently plotting $\Delta M$ against $1 / \kappa_{c}$ and interpolating to $\Delta M=0$ gives $\kappa_{c}^{*}$. 


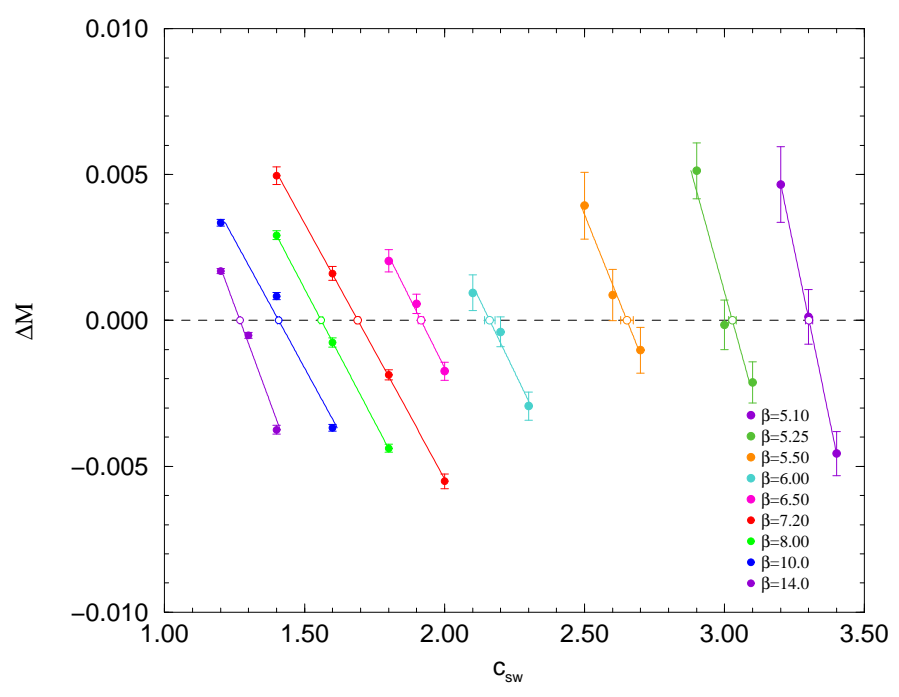

Figure 2: $\Delta M$ at $M=0$ against $c_{s w}$ for various values of $\beta$ (filled circles) together with linear interpolations to $\Delta M=0$ (open circles).

\section{Results}

The results for $c_{s w}^{*}$ and $\kappa_{c}^{*}$ against $g_{0}^{2}$ are plotted in Figs. 3, 46 4 respectively in the range $\beta \leq 5.10$.

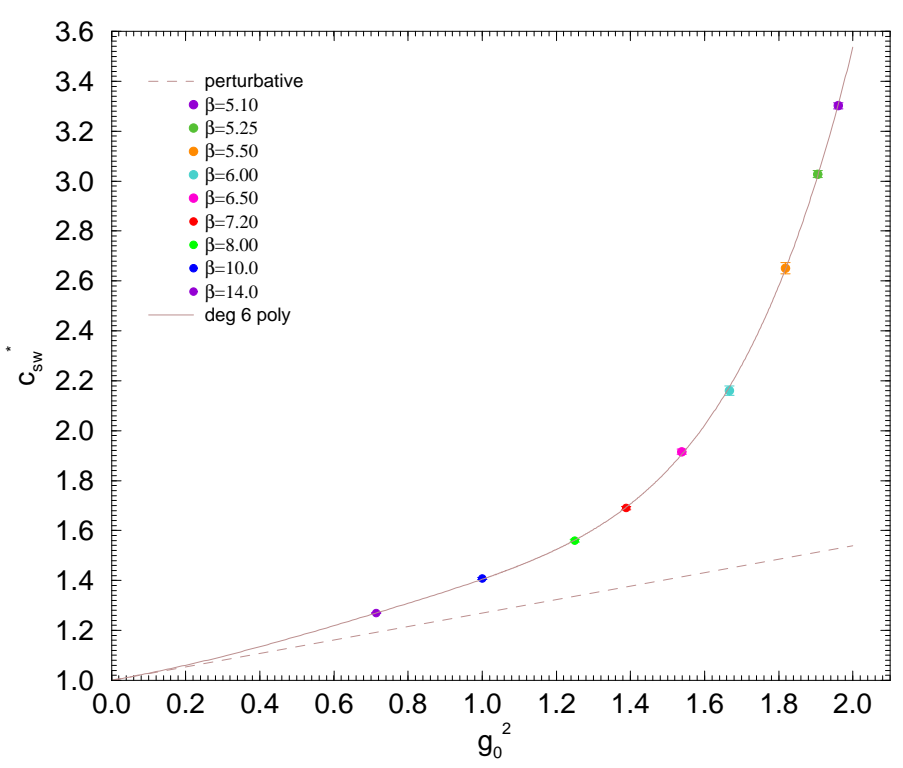

Figure 3: $c_{s w}^{*}$ against $g_{0}^{2}$ for various values of $\beta$ (circles), together with a polynomial interpolation (line). Also shown is the perturbative result.

The lowest order perturbative limit has been computed for both $c_{s w}^{*}$ and $\kappa_{c}^{*}$, [3] and is also shown in the figures. An interpolation between the numerically determined points is also shown. For both $c_{s w}^{*}$ and $\kappa_{c}^{*}$ a 6 th order polynomial in $g_{0}^{2}$ proved sufficient. (These interpolation functions are constrained to reproduce the perturbative results, [3] , in the $\beta \rightarrow \infty$ limit. Therefore, they have four 


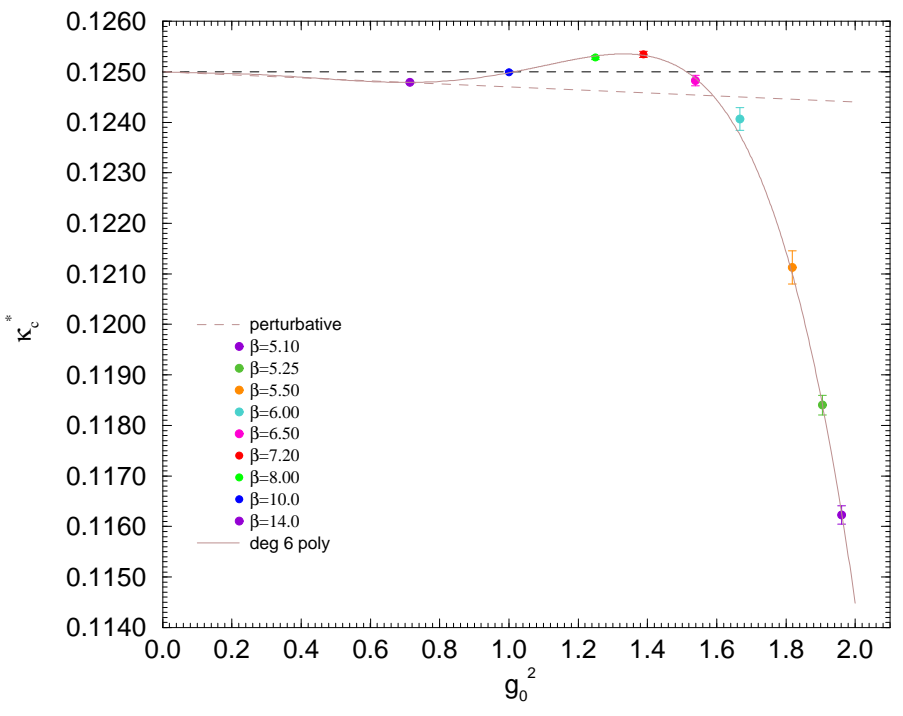

Figure 4: $\kappa_{c}^{*}$ against $g_{0}^{2}$ for various values of $\beta$ (circles), together with a polynomial interpolation (line). Also shown is the perturbative result.

free parameters.) This smooth fit between the points gives an estimate for $c_{s w}^{*}$ which will be used in the action for future generation of configurations.

For $c_{s w}^{*}$ the polynomial only tracks the perturbative solution for small values of $g_{0}^{2}$. This is perhaps not surprising as the tadpole improved, $T I$, estimate is $c_{s w}^{T I}=u_{0}^{(S)} / u_{0}^{4}$, [阿, which is to be compared with the unsmeared case of $c_{s w}^{T I}=1 / u_{0}^{3}$ where $u_{0}$ is the average plaquette value and $u_{0}^{(S)}$ is the smeared value. As smearing increases the plaquette value this indicates that $c_{s w}^{*}$ can be large. For $\kappa_{c}^{*}$ on the other hand as $\kappa_{c}^{T I}=1 /\left(8 u_{0}^{(S)}\right)$ we expect that it is $\sim 1 / 8$. This is true for reasonably fine lattices, however $\kappa_{c}^{*}$ does begin to decrease for larger values of $g_{0}^{2}$. For $n_{f}=2$ the same phenomenon occurs: for larger $g_{0}^{2}, \kappa_{c}^{*}$ begins to decrease (after initially increasing).

Finally in Fig. 5 we show the vector renormalisation constant. This is computed using the vector current in the ratio of a three-point to two-point function where the sinks/sources are built using $O^{(i)}$ as described earlier in section 1 .

\section{Conclusions}

Non-perturbative $O(a)$ improvement is a viable procedure for (stout) smeared actions with typical clover results being obtained. (Other recent results for $2+1$ flavours are given in [6, 77.) As $a$ decreases we need a significant $c_{s w} \gg c_{s w}^{\text {tree }} \equiv 1$ for $O(a)$ improvement. We are now seeking a region where $a \sim 0.05-0.1 \mathrm{fm}$. Improvement, which is presumably an asymptotic series, brings an advantage for smaller $a$ say $a \leq 0.1 \mathrm{fm}$. The two extremes for $a$ are simulations at small $a$ with 'large' $m_{p s}$ when there is no continuum extrapolation but a chiral extrapolation, or alternatively simulations at 'coarse' $a$ with $m_{p s} \sim m_{\pi}$ when there is no chiral extrapolation but a continuum extrapolation. Of course the Schrödinger functional does not tell us $a$; for this conventional HMC simulations are required. Some preliminary results indicate that around $\beta \lesssim 6.0$ we have $a \lesssim$ $0.07 \mathrm{fm}$. Final results, including larger lattice size comparisons will be published elsewhere, [8]. 


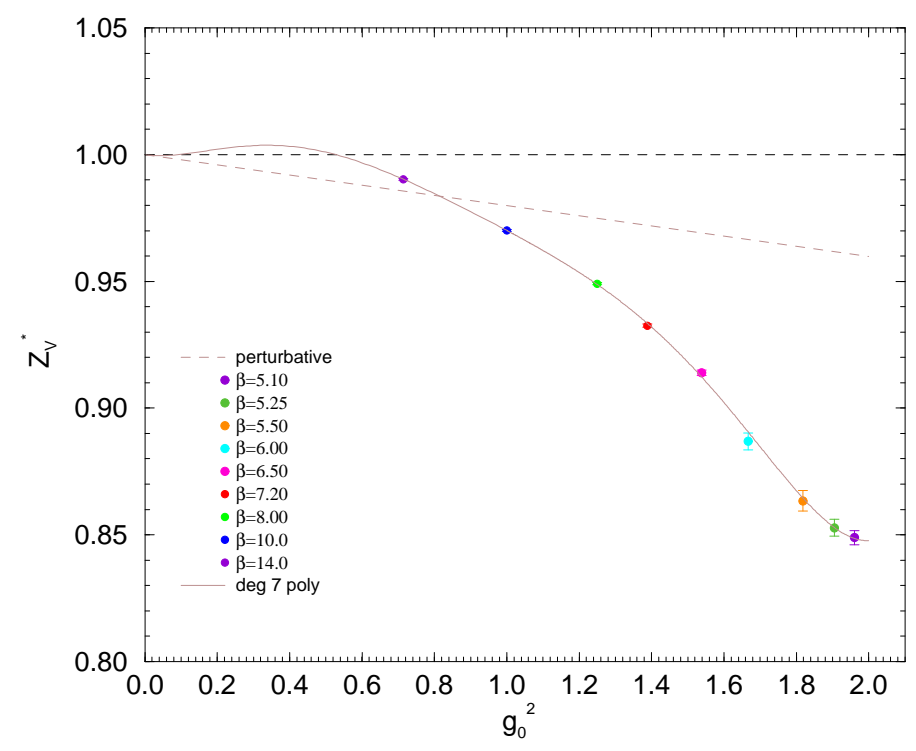

Figure 5: $Z_{V}^{*}$ against $g_{0}^{2}$ for various values of $\beta$ (circles), together with a polynomial interpolation (line). Also shown is the perturbative result.

\section{Acknowledgements}

The numerical calculations have been performed on the BlueGeneLs at EPCC (Edinburgh, UK), NIC (Jülich, Germany), the QCDOC (Edinburgh, UK) and the SGI ICE at HLRN (BerlinHannover, Germany). The BlueGene and QCDOC codes were optimised using Bagel, [9]. This work has been supported in part by the EU Integrated Infrastructure Initiative Hadron Physics (I3HP) under contract RII3-CT-2004-506078 and by the DFG under contracts FOR 465 (Forschergruppe Gitter-Hadronen-Phänomenologie) and SFB/TR 55 (Hadron Physics from Lattice QCD).

\section{References}

[1] M. Lüscher et al., Nucl. Phys. B384, 168 (1992) [arXiv: hep-lat/9207009]; Nucl. Phys. B478, 365 (1996) [arXiv: hep-lat/9605038].

[2] M. Lüscher et al., Nucl. Phys. B491, 323 (1997) [arXiv: hep-lat/9609035].

[3] R. Horsley et al., QCDSF Collaboration, Phys. Rev. D78, 054504 (2008) arXiv : 0807 . 034 5; H. Perlt, QCDSF Collaboration, talk at Lattice 2008.

[4] R. Edwards and B. Joó, Nucl. Phys. Proc. Suppl. 140, 832 (2005) [arXiv: hep-lat/ 0409003 ].

[5] T. Klassen, Nucl. Phys. B509, 391 (1998) [arXiv: hep-lat/9705025].

[6] N.Yamada, et al., CP-PACS, JLQCD Collaborations, Phys. Rev. D71, 054505 (2005) [arXiv: hep-lat/ 0406028 ]; S. Aoki, et al., CP-PACS, JLQCD Collaborations, Phys. Rev. D73, 034501 (2006) [arXiv: hep-lat/0508031].

[7] R. G. Edwards et al., arXiv:0803.3960.

[8] N. Cundy et al., QCDSF-UKQCD Collaborations, in preparation.

[9] P. A. Boyle, http://www.ph.ed.ac.uk/ paboyle/Bagel.html (2005). 